\title{
UNIFORM SPACES AS NICE IMAGES OF NICE UNIFORM AND METRIC SPACES ${ }^{(1)}$
}

\author{
BY \\ RICHARD WILLMOTT
}

1. Introduction. The classical theorem that a complete separable metric space is the image under a one-to-one continuous function of a closed subset of the irrational numbers has been extended in two directions, the first leading to various characterizations in descriptive set theory of Borel and analytic sets or generalizations of them as continuous images of certain subsets of the irrationals, or generalizations of them (see, e.g. [3] and references cited there; [4]; [6]). The second direction originates in the observation that a closed subset of the irrationals is a complete 0 -dimensional metric space (under a suitable metric), and leads to the general question asked by Alexandroff [1], "Which spaces can be represented as images of 'nice' (e.g. metric, 0-dimensional) spaces under 'nice' [e.g. one-to-one, open, closed, perfect] continuous mappings?' (See, e.g. [7], [9] and the survey articles [1], [2] and [11].)

Two representative results in this area are the following theorems. (The weight of a topological space is the least cardinal of a base for the topology.)

TheOREM A (Stone [14], Lemma 3.3, corollary and observations in Section 2). If $X$ is a complete metric space of weight $\leq \boldsymbol{N}_{\alpha}$, then $X$ is the image of a complete, 0-dimensional metric space of weight $\leq \boldsymbol{\aleph}_{\alpha}$ under a function which is continuous, one-to-one and is such that the image of each open set is a countable union of closed sets.

THEOREM B (Ponomarev [10]). If $X$ is a normal space, then $X$ is the image of a normal 0-dimensional space of the same weight under a function which is continuous, closed and is such that the inverse image of every point is compact.

In this paper we first establish the result (contained in Theorem 2), typical of the first direction indicated above that a complete uniform space (with a certain cardinality restriction) is the image, under a continuous one-to-one function, of a closed subset of a certain space (which is a generalization of the irrationals). It follows from this theorem or one of Kruse [6] that (see Theorems 3 and 4) $a$ complete uniform space (with a certain cardinality restriction) is the image,

Received by the editors February 22, 1978 and, in revised form, October 2, 1978.

(1) This work was supported by the National Research Council of Canada. 
under a continuous one-to-one function, of a complete 0-dimensional uniform space of the same or smaller weight, a result in the second direction in the same spirit as Theorems A and B. Finally we use the fact that the domain space in the above result may be taken to be a metric space and obtain in Theorems 5 and 6: a complete uniform space (with a certain cardinality restriction) is the image, under a continuous one-to-one function of a complete 0-dimensional metric space of the same or related weight (depending on some other cardinality conditions), a theorem again in the direction of Theorems A and B but in which the domain space has a nicer property (metric) than the given space (uniform).

In much of the work in the direction indicated by Alexandroff, including Theorems A and B, the nice space, particularly in the case of 0 -dimensionality, has been a subset of a product of discrete spaces. When these products, which include the generalizations of the irrationals called the Baire spaces, are taken over more than countably many factors, they are not metrizable. We work with a second generalization of the irrationals which under certain conditions (occurring for arbitrarily high cardinals) gives a metrizable space (see Section 3).

I am indebted to A. H. Kruse for comments and suggestions on aspects of this paper.

2. Preliminaries. We adopt a development of ordinal numbers in which each ordinal is just the set of all preceding ordinals. (For notational convenience, cardinals are not identified with initial ordinals). Lower case Greek letters will be used to denote ordinals and, as usual, $\omega$ will denote the function on the ordinals such that $\omega_{\alpha}$ is the least ordinal of cardinal $\aleph_{\alpha} . \omega_{0}$ is thus the set of nonnegative integers. An ordinal number $\omega_{\alpha}$ is cofinal with $\omega_{0}$ if $\omega_{\alpha}=\bigcup_{n \in \omega_{0}} p_{n}$ for some strictly increasing sequence $\left\{p_{n}\right\}$ of elements of $\omega_{\alpha} . \omega_{\alpha}$ is cofinal with $\omega_{0}$ if and only if $\alpha=0$ or is cofinal with $\omega_{0}$. For any ordinal $\gamma, \gamma+\omega_{0}$ is cofinal with $\omega_{0}$.

The pair of cardinals $\left(\aleph_{\alpha}, \aleph_{\beta}\right)$ will be said to have property $E$ if for every non-zero cardinal $k<\aleph_{\beta}, \aleph_{\alpha}^{k}=\aleph_{\alpha}$. For such a pair, of course, $\alpha \geq \beta$. We remark that if $\alpha \neq 0$ is not a limit ordinal, then since $\aleph_{\alpha}^{\aleph_{\alpha-1}}=2^{\aleph_{\alpha-1}}$, we have that $\left(\aleph_{\alpha}, \aleph_{\alpha}\right)$ has property $E$ if and only if $\aleph_{\alpha}=2^{\aleph_{\alpha-1}}$. Thus GCH implies that $\left(\aleph_{\alpha}, \aleph_{\alpha}\right)$ has property $E$ if $\alpha$ is not a limit ordinal, while, for example, $\left(\aleph_{\alpha}, \aleph_{\alpha}\right)$ fails to have property $E$ if $\alpha$ is cofinal with $\omega_{0}\left([8]\right.$, Theorem 23.8). $\left(\aleph_{\alpha}, \aleph_{0}\right)$ of course has property $E$ for any $\aleph_{\alpha}$.

We follow Kruse ([6], p. 11) in making the following definitions. (For uniformity notation and concepts see Kelley [5], Chapter 6.) A uniform space $(X, \mathcal{U})$ is uniformly $\aleph_{\alpha}$-Lindelof if for each $U \in \mathcal{U}$, the cover $\{U[\{x\}]: x \in X\}$ of $X$ has a subcover of at most $\aleph_{\alpha}$ elements. An $(\alpha, \beta)$-space is a complete uniformly $\aleph_{\alpha}$-Lindelof uniform space $(X, \mathcal{U})$ such that $\mathcal{U}$ has a base of cardinal 
$\leq \aleph_{\beta}$ and $X$ with the uniform topology is Hausdorff. Any closed subset of an $(\alpha, \beta)$-space is again an $(\alpha, \beta)$-space under the relative uniformity.

Although we do not specify that $\aleph_{\alpha}$ and $\aleph_{\beta}$ be the least cardinals with the stated properties, even if they were, it would not necessarily follow that $\aleph_{\alpha} \geq \aleph_{\beta}$. For example, any non-first countable compact Hausdorff space would be Lindelof $\left(\aleph_{0}\right.$-Lindelof) and would have a minimum cardinal $\aleph_{\beta}$ exceeding $\aleph_{0}$. It is easy to verify, using an extension of the usual argument that a Lindelof metric space is second countable, that if $\alpha \geq \beta$, the weight of an $(\alpha, \beta)$-space is at most $\aleph_{\alpha}$.

3. The spaces $(I(\alpha, \beta), \mathscr{P})$ and $(I(\alpha, \beta), \Im)$. Let $I(\alpha, \beta)=\omega_{\alpha}^{\omega_{\beta}}$, which can be considered as the $\omega_{\beta}$-fold product of the set $\omega_{\alpha}$ with itself or as the set of all transfinite sequences of length $\omega_{\beta}$ with values in $\omega_{\alpha}$. Using the first interpretation we will denote by $\mathscr{P}$ the product topology on $I(\alpha, \beta)$ when $\omega_{\alpha}$ is given the discrete topology. $(I(\alpha, \beta), \mathscr{P})$ is an $(\alpha, \beta)$-space ([6], Prop. 4.5$)$; it is metrizable if and only if $\beta=0$; its weight is $\max \left\{\aleph_{\alpha}, \aleph_{\beta}\right\}$; and it is 0 -dimensional (in the sense of small inductive dimension ind). This space was introduced and used extensively by Kruse [6] (whose notation is different). In the case $\beta=0$, it is the standard Baire space $B\left(\aleph_{\alpha}\right)$ (see, e.g. [14]).

Considering now $I(\alpha, \beta)$ as a space of sequences, for $i \in I(\alpha, \beta), \gamma \in \omega_{\beta}$, the restriction of $i$ to $\gamma, i \mid \gamma$ is an ordered $\gamma$-tuple of elements of $\omega_{\alpha}$, i.e. a function on $\gamma$ to $\omega_{\alpha}$. For $i \in I(\alpha, \beta), \gamma \in \omega_{\beta}$, we define

$$
I(i \mid \gamma)=\{j \in I(\alpha, \beta): j|\gamma=i| \gamma\}
$$

To define a topology $\mathfrak{I}$ on $I(\alpha, \beta)$ we take as a base for the neighborhood system of a point $i$ the set of all $I(i \mid \gamma), \gamma \in \omega_{\beta}$. That is, $\Im=\{G \subset I(\alpha, \beta)$ : if $i \in G$, then for some $\left.\gamma \in \omega_{\beta}, I(i \mid \gamma) \subset G\right\}$. The space $(I(\alpha, \beta), \mathfrak{\Im})$ is a special case of spaces studied in [13], where it is shown that such a space is normal, regular, $T_{1}$, totally disconnnected, perfect, separable if and only if $\alpha=\beta=0$, and metrizable if $\beta=0$. A slight extension of the proof of the last assertion shows that it is metrizable also if $\beta$ is cofinal with $\omega_{0}$. This property occurs for arbitrarily high ordinals, as $\gamma+\omega_{0}$ is cofinal with $\omega_{0}$ for any ordinal $\gamma$. It is easy to check that the sets $I(i \mid \gamma)$ are open and closed ([13], Lemma 2), homeomorphic to $(I(\alpha, \beta), \mathfrak{\Im})$, and that any two of them are either disjoint, or one is contained in the other ([13], Lemma 1). Clearly, too, if $\gamma<\omega_{\beta}$, the set of $i \in I(\alpha, \beta)$ with any particular ones of the coordinates up to $\gamma$ fixed is open and closed. It follows readily from Theorem 4, Section 2, Chapter 6, p. 250 of [4], and the fact that the elements $I(i \mid \gamma)$ of a base for the topology are open and either disjoint or nested, that $(I(\alpha, \beta), \mathfrak{I})$ is strongly 0 -dimensional and hence ([4], Section 1, Chapter 7, comments following the definitions) has small inductive (ind), large inductive and covering dimension 0. 
If for each $\gamma \in \omega_{\beta}$ we let

$$
U_{\gamma}=\bigcup_{i \in I(\alpha, \beta)} I(i \mid \gamma) \times I(i \mid \gamma)
$$

and then let $\mathscr{B}=\left\{U_{\gamma}: \gamma \in \omega_{\beta}\right\}$, it follows easily that $\mathscr{B}$ is a base for a uniformity $\mathcal{U}$ on $I(\alpha, \beta)$ whose topology is $\Im$. As $\mathscr{B}$ is a nested well-ordered base for $\mathcal{U}$, transfinite sequences of length $\omega_{\beta}$ are sufficient to handle convergence in $(I(\alpha, \beta), \mathcal{U})$ and it is clear that $(I(\alpha, \beta), \mathcal{U})$ is complete, i.e. every Cauchy transfinite sequence of length $\omega_{\beta}$ converges to a point in $I(\alpha, \beta)$. (Loosely speaking, the points (sequences) in a Cauchy transfinite sequence in $I(\alpha, \beta)$ just have more and more coordinates fixed.)

The sets $I(i \mid \gamma)$ form a base for $\Im$ and there are $\sum_{\gamma \in \omega_{\beta}} \aleph_{\alpha}^{|\gamma|}$ of them (where $|\gamma|$ is the cardinality of $\gamma)$. Thus if $\left(\aleph_{\alpha}, \aleph_{\beta}\right)$ has property $E,(I(\alpha, \beta), \mathfrak{I})$ has weight $\leq \aleph_{\alpha}$, and since for any $i \in I(\alpha, \beta)$ and $\gamma \in \omega_{\beta}, U_{\gamma}[\{i\}]=I(i \mid \gamma)$, we have that $(I(\alpha, \beta), \mathcal{U})$ is uniformly $\aleph_{\alpha}$-Lindelof and is hence an $(\alpha, \beta)$-space.

The space $(I(\alpha, \beta), \Im)$ was also introduced by Kruse [6], but was not much studied or used there. It and $(I(\alpha, \beta), \mathscr{P})$ are both generalizations of the irrationals $I$ between 0 and 1 , for $(I(0,0), \Im),(I(0,0), \mathscr{P})$ and $I$ with the usual topology are homeomorphic. We remark that while $(I(\alpha, 0), \Im)$ and $(I(\alpha, 0), \mathscr{P})$ are homeomorphic, $\mathfrak{I}$ strictly contains $\mathscr{P}$ on $I(\alpha, \beta)$ if $\beta>0$.

4. The image theorems. The first two theorems are similar in form, but differ somewhat in aspects of the hypotheses and conclusions. The first is due to Kruse ([6], Theorem 15.13) who works with $(I(\alpha, \beta), \mathscr{P})$, while the second uses $(I(\alpha, \beta), \mathfrak{\Im})$. If $\mathscr{F}$ is the family of closed sets of some topological space, Borel $(\alpha) \mathscr{F}$ is the smallest family of sets containing $\mathscr{F}$ and closed under complementation and unions of $\aleph_{\alpha}$ elements of $\mathscr{F}$.

TheOREM 1. Suppose $\alpha \geq \gamma \geq \beta$ and $X$ is an $(\alpha, \beta)$-space. If $B$ is a Borel $(\gamma) \mathscr{F}$-set in $X$, then $B$ is the image, under a continuous one-to-one function, of a closed subset of $(I(\alpha, \gamma), \mathscr{P})$.

THEOREM 2. Suppose $X$ is an $(\alpha, \beta)$-space, $\alpha \geq \beta$, and $\left(\aleph_{\alpha}, \aleph_{\alpha}\right)$ has property $E$. If $B$ is the union of $\aleph_{\alpha}$ closed sets in $X$, then $B$ is the image, under $a$ continuous one-to-one function, of a closed subset of $(I(\alpha, \beta), \mathfrak{\Im})$.

Proof. The proof is based on the idea in a proof of Rogers ([12], Lemma 2). Let $\left\{U_{\gamma}: \gamma \in \omega_{\beta}\right\}$ be a base for a uniformity for $X$ consisting of closed symmetric members of the uniformity ([5], Theorem 6.8). Given $\gamma \in \omega_{\beta}$, choose $v$ so that $U_{\nu} \circ U_{\nu} \subset U_{\gamma}$, and then by the uniformly $\aleph_{\alpha}$-Lindelof property, choose $\aleph_{\alpha}$ sets of the form $U_{\nu}[\{x\}]$ which cover $X . X$ is then the union of $\aleph_{\alpha}$ closed sets each of whose cross product with itself is contained in $U_{\gamma}$. We have then, for each $\gamma \in \omega_{\beta}$,

$$
X=\bigcup_{\delta \in \omega_{\alpha}} A(\gamma, \delta)
$$


where for each $\delta \in \omega_{\alpha}, A(\gamma, \delta) \times A(\gamma, \delta) \subset U_{\gamma}$, and $A(\gamma, \delta)$ is closed. By taking intersections with the $\aleph_{\alpha}$ closed sets of the form $A(0, \delta)$, we may express $B$ as a union of $\aleph_{\alpha}$ closed sets $B=\bigcup_{\gamma \in \omega_{\alpha}} B(\gamma)$, where for each $\gamma \in \omega_{\alpha}, B(\gamma) \times B(\gamma) \subset$ $U_{0}$. We now form systems of sets $F(i \mid \gamma), i \in I(\alpha, \beta), \gamma \in \omega_{\beta}$. Let $F$ (empty sequence $)=X$. For each $i \in I(\alpha, \beta)$ let $F(i \mid 1)=B(i \mid 1)=B(i(0))$. Consider any $\eta \in \omega_{\beta} \backslash 2$. Having defined closed sets $F(i \mid \gamma)$ for all $i \in I(\alpha, \beta)$ and all $\gamma<\eta$ such that if $\gamma$ is not a limit ordinal or the successor of a limit ordinal, $F(i \mid \gamma) \times$ $F(i \mid \gamma) \subset U_{\gamma}$, we now define the sets $F(i \mid \eta), i \in I(\alpha, \beta)$ as follows, distinguishing two cases.

(1) $\eta$ a limit ordinal or the successor of a limit ordinal. Let $F(i \mid \eta)=X$ for all $i \in I(\alpha, \beta)$.

(2) $\eta$ not a limit ordinal or the successor of a limit ordinal.

We introduce some notation. If $h$ is a function on the ordinal $\gamma$, then $h ; \delta$ denotes $h \cup\{(\gamma, \delta)\}$, that is, the function on $\gamma+1$ that agrees with $h$ on the subset $\gamma$ of $\gamma+1$, and takes the value $\delta$ at the element $\gamma$ of $\gamma+1$. Thus, for example, if $\gamma$ is not a limit ordinal, $(j \mid \gamma)=(j \mid \gamma-1 ; j(\gamma-1))$. Suppose $j \in$ $I(\alpha, \beta)$. The set

$$
\bigcup_{\delta<j(\eta-2)} F(j \mid \eta-2 ; \delta)
$$

is a union of $\leq|j(\eta-2)|$ closed sets, where $|j(\eta-2)|<\aleph_{\alpha}$ since $j(\eta-2) \in \omega_{\alpha}$. Now if $F$ is closed, then for each $\gamma \in \omega_{\beta}$ there exists open $G_{\gamma}$, with $F \subset G_{\gamma} \subset$ $U_{\gamma}[F]$ and so

$$
F \subset \bigcap_{\gamma \in \omega_{\beta}} G_{\gamma} \subset \bigcap_{\gamma \in \omega_{\beta}} U_{\gamma}[F]=F
$$

and $F$ is the intersection of $\leq \boldsymbol{\aleph}_{\beta}$ open sets. Thus the difference set

$$
X \sim \bigcup_{\delta<j(\eta-2)} F(j \mid \eta-2 ; \delta)
$$

is the intersection of $\leq|j(\eta-2)|$ open sets, each of which is the union of $\leq \boldsymbol{\aleph}_{\beta}$ closed sets. Hence for some function $E$ on $j(\eta-2) \times \omega_{\beta}$ to closed sets,

$$
\begin{aligned}
X \sim \bigcup_{\delta<j(\eta-2)} F(j \mid \eta-2 ; \delta) & =\bigcap_{\rho \in j(\eta-2)} \bigcup_{\gamma \in \omega_{\beta}} E(\rho, \gamma) \\
& =\bigcup_{h \in \omega_{\beta} j(\eta-2)} \bigcap_{\rho \in j(\eta-2)} E(\rho, h(\rho)),
\end{aligned}
$$

the second equality following from a standard general distributive law (see e.g. the proof of Lemma 4.8 in [3]). The last expression is a union over $\aleph_{\beta}^{l j(\eta-2) \mid}$ closed sets, where $\aleph_{\beta}^{|j(\eta-2)|} \leq \aleph_{\alpha}$, since $\aleph_{\beta} \leq \aleph_{\alpha},|j(\eta-2)|<\aleph_{\alpha}$ and $\left(\aleph_{\alpha}, \aleph_{\alpha}\right)$ has property $E$. We enumerate these sets as follows, using the empty set if necessary.

$$
X \sim \bigcup_{\delta<j(\eta-2)} F(j \mid \eta-2 ; \delta)=\bigcup_{\gamma \in \omega_{\alpha}} F(j \mid \eta-1 ; \gamma)
$$


We may assume that the sets $F(j \mid \eta-1 ; \gamma)$ are such that $F(j \mid \eta-1 ; \gamma) \times$ $F(j \mid \eta-1 ; \gamma) \subset U_{\eta}$, for otherwise we may take intersections with the $\aleph_{\alpha}$ closed sets of the form $A(\eta, \delta)$ giving still at most $\aleph_{\alpha} \cdot \aleph_{\alpha}=\aleph_{\alpha}$ closed sets. Carrying out this process for all $j \in I(\alpha, \beta)$ yields sets $F(i \mid \eta)$ for all $i \in I(\alpha, \beta)$, and thus the sets $F(i \mid \gamma)$ for all $i \in I(\alpha, \beta), \gamma \in \omega_{\beta}$ are defined by transfinite induction.

Now for $i \in I(\alpha, \beta)$ set

$$
F(i)=\bigcap_{\gamma \in \omega_{\beta}} F(i \mid \gamma)
$$

and since, for $\gamma$ not a limit ordinal or the successor of a limit ordinal, $F(i \mid \gamma) \times F(i \mid \gamma) \subset U_{\gamma}$, it follows that $F(i)$ is a singleton or is empty. Let $J=\{i \in I(\alpha, \beta): F(i) \neq \varnothing\}$ and define a function $f$ on $J$ to $B$ by taking $f(i) \in F(i)$ for $i \in J$. We check that $f$ is a one-to-one continuous function on $J$ onto $B$, and that $J$ is closed in $(I(\alpha, \beta), \Im)$.

(a) $f$ is one-to-one. Suppose $i, j \in J, i \neq j$. Then for some $\eta \in \omega_{\beta}, i(\gamma)=j(\gamma)$ for $\gamma<\eta$ and (say) $i(\eta)<j(\eta)$. Then

$$
\begin{aligned}
F(j \mid \eta+2) \subset X \sim \bigcup_{\delta<j(\eta)} F(j \mid \eta ; \delta) \subset X \sim F(j \mid \eta ; i(\eta)) & \\
& =X \sim F(i \mid \eta ; i(\eta))=X \sim F(i \mid \eta+1) .
\end{aligned}
$$

Hence $F(j \mid \eta+2) \cap F(i \mid \eta+1)=\varnothing$, so $F(j) \cap F(i)=\varnothing$ and $f(i) \neq f(j)$.

(b) $f$ is onto $B$. Suppose $x \in B$. Let $i(0)$ be the least element of $\omega_{\alpha}$ such that $x \in F(i \mid 1)=F(i(0))=B(i(0))$. Then $x \notin \bigcup_{\delta<i(0)} F(\delta)$ so

$$
x \in X \sim \bigcup_{\delta<i(0)} F(\delta)=\bigcup_{\gamma \in \omega_{\alpha}} F(i(0), \gamma),
$$

and hence for some $j \in I(\alpha, \beta)$ such that $j(0)=i(0), x \in F(j \mid 2)$. In general, suppose that for every $\gamma<\eta, x \in F(i \mid \gamma+1)$ and $i(\gamma)$ is the least element of $\omega_{\alpha}$ for which this is true. We have two cases.

(1) If $\eta$ is a limit ordinal, then $x \in F(i \mid \eta)=F(i \mid \eta ; 0)=X$, and we take $i(\eta)=0$.

(2) If $\eta$ is not a limit ordinal, then

and so

$$
x \notin \bigcup_{\delta<i(\eta-1)} F(i \mid \eta-1 ; \delta)
$$

$$
x \in X \sim \bigcup_{\delta<i(\eta-1)} F(i \mid \eta-1 ; \delta)=\bigcup_{\gamma \in \omega_{\alpha}} F(i \mid \eta ; \gamma) .
$$

Hence $x \in F(j \mid \eta+1)$ for some $j \in I(\alpha, \beta)$ such that for $\gamma<\eta, j|\gamma+1=i| \gamma+1$. It follows that for some $i \in I(\alpha, \beta), x \in F(i \mid \gamma)$ for every $\gamma \in \omega_{\beta}$, so that

$$
x \in \bigcap_{\gamma \in \omega_{\beta}} F(i \mid \gamma)=F(i)
$$

and $x=f(i)$. 
(c) $f$ is continuous. Suppose $i \in J, G^{\prime}$ is open in $B$ and $f(i) \in G^{\prime}$. Let $G$ be open in $X, G^{\prime}=G \cap B$. Then for some $U$ in the uniformity for $X, f(i) \in$ $U[f(i)] \subset G$. Choose $\gamma \in \omega_{\beta}$ such that $F(i \mid \gamma) \times F(i \mid \gamma) \subset U$. Then $F(i \mid \gamma) \subset$ $U[f(i)] \subset G$, for if $y \in F(i \mid \gamma)$, then $(f(i), y) \in F(i \mid \gamma) \times F(i \mid \gamma) \subset U$ and so $y \in$ $U[f(i)]$.

Now suppose $k \in J \cap I(i \mid \gamma)$, a neighborhood of $i$ in $J$. Then $f(k) \in$ $\bigcap_{\eta \in \omega_{\beta}} F(k \mid \eta) \subset F(k \mid \gamma) \cap B=F(i \mid \gamma) \cap B \subset G \cap B=G^{\prime}$, showing that $f$ is continuous.

(d) $J$ is closed in $(I(\alpha, \beta)$, Э). Suppose $j$ is a limit point of $J$. Then for each $\gamma \in \omega_{\beta}$ there exists $i^{\gamma} \in J$ such that $i^{\gamma}|\gamma=j| \gamma$. Hence for each $\eta \in \omega_{\beta}$,

$$
F_{\eta}=\bigcap_{\gamma \leq \eta} F(j \mid \gamma)=\bigcap_{\gamma \leq \eta} F\left(i^{\eta} \mid \gamma\right) \neq \varnothing .
$$

The sets $F_{\eta}$ are closed; if $\eta$ is not a limit ordinal or the successor of a limit ordinal, $F_{\eta} \times F_{\eta} \subset U_{\eta}$; and if $\eta<\gamma \in \omega_{\beta}, F_{\gamma} \subset F_{\eta}$. It follows by completeness ([5], Theorem 6.23 , p. 193) that

$$
F(j)=\bigcap_{\gamma \in \omega_{\beta}} F(j \mid \gamma)=\bigcap_{\gamma \in \omega_{\beta}} F_{\gamma} \neq \varnothing .
$$

Hence $j \in J$, and $J$ is closed. This completes the proof.

Remarks. In the circumstances of Theorem $2, \beta=\alpha$, then each open set $B$ is a union of $\aleph_{\alpha}$ closed ones and so satisfies the requirements of the theorem.

If $\alpha \geq \beta$ and $\left(\aleph_{\alpha}, \aleph_{\alpha}\right)$ has property $E$, then so has $\left(\aleph_{\alpha}, \aleph_{\beta}\right)$ and a closed subset of $(I(\alpha, \beta), \Im)$ is an $(\alpha, \beta)$-space and so by Theorem 1 (with $\gamma=\beta)$ is the image, under a continuous one-to-one function of a closed subset of $(I(\alpha, \beta), \mathscr{P})$. Thus Theorem 2 remains valid if $\Im$ is replaced by $\mathscr{P}$. As a subset of $I(\alpha, \beta)$ closed under $\mathscr{P}$ is necessarily closed under $\mathfrak{S}$, Theorem 1 remains valid if $\mathscr{P}$ is replaced by $\mathfrak{\Im}$.

Properties of the $I(\alpha, \beta)$ spaces are now used to obtain as corollaries to the above two theorems image theorems analogous to Theorems A and B of the introduction.

Theorem 3. Suppose that $X$ is an $(\alpha, \beta)$-space, $\alpha \geq \gamma \geq \beta$ and $B$ is a Borel $(\gamma) \mathscr{F}$-set in $X$. Then $B$ is the image, under a continuous, one-to-one function of a 0 -dimensional (in the sense of small inductive dimension ind) $(\alpha, \beta)$-space of weight $\leq \aleph_{\alpha}$.

Proof. The closed subset of $(I(\alpha, \beta), \mathscr{P})$ obtained in Theorem 1 is again an $(\alpha, \beta)$-space and so satisfies the requirements of this theorem, as any subset of a regular 0-dimensional space is 0 -dimensional ([4], Theorem 1, Section 1, Chapter 7, p. 264).

TheOREM 4. Suppose that $X$ is an $(\alpha, \beta)$-space, $\alpha \geq \beta,\left(\aleph_{\alpha}, \aleph_{\alpha}\right)$ has property $E$ and $B$ is the union of $\aleph_{\alpha}$ closed sets in $X$. Then $B$ is the image, under $a$ 
continuous one-to-one function, of a 0 -dimensional (in any sense) $(\alpha, \beta)$-space of weight $\leq \aleph_{\alpha}$.

Proof. Since $\alpha \geq \beta$ and $\left(\aleph_{\alpha}, \aleph_{\alpha}\right)$ has property $E$, so does $\left(\aleph_{\alpha}, \aleph_{\beta}\right)$; so $(I(\alpha, \beta), \mathcal{U})$ is an $(\alpha, \beta)$-space of weight $\aleph_{\alpha}$. Again the closed subset of $(I(\alpha, \beta), \Im)$ obtained in Theorem 2 has the properties desired, as for normal spaces strong 0 -dimensionality is hereditary with respect to closed sets ([4], Theorem 7, Section 2, Chapter 6, p. 251).

We now employ the fact that there are spaces $(I(\alpha, \beta), \mathfrak{s})$ for arbitrarily high $\beta$ which are metrizable to get our given uniform spaces as images of metrizable ones.

Theorem 5. Suppose that $X$ is an $(\alpha, \beta)$-space, $\alpha \geq \gamma \geq \beta, B$ is a Borel $(\gamma) \mathscr{F}$-set in $X$, and either

(i) $\gamma$ is cofinal with $\omega_{0}$; or

(ii) $\alpha \geq \gamma+\omega_{0}=\nu$; or

(iii) $\alpha<\gamma+\omega_{0}=\nu$.

Then $B$ is the image, under a continuous one-to-one function, of a complete 0 -dimensional (in any sense) metric space of weight respectively:

(i) $\leq \sum_{\delta \in \omega_{\gamma}} \aleph_{\alpha}^{|\delta|}$.

(ii) $\leq \sum_{\delta \in \omega_{\nu}} \aleph_{\alpha}^{|\delta|}$.

(iii) $\leq \sum_{\delta \in \omega_{\nu}} \aleph_{\nu}^{|\delta|}$.

Proof. An $(\alpha, \beta)$-space is also a $(\delta, \nu)$-space if $\delta \geq \alpha, \nu \geq \beta$, and since $\mathscr{P} \subset \Im$, a closed subset of any space $(I(\alpha, \beta), \mathscr{P})$ is also closed in $(I(\alpha, \beta), \mathfrak{\Im})$. By these facts and Theorem 1 , then, $B$ is the image, under a continuous one-to-one function of a closed subset of $(I(\alpha, \gamma), \Im),\left(I\left(\alpha, \gamma+\omega_{0}\right), \Im\right)$ and $\left(I\left(\gamma+\omega_{0}, \gamma+\right.\right.$ $\left.\omega_{0}\right), \mathfrak{\Im}$ ) in cases (i), (ii) and (iii) respectively. These spaces are 0 -dimensional complete metric spaces satisfying the stated restrictions on weight, so the same is true of closed subsets.

The additional restrictions in the final theorem are due to the fact that if $\left(\aleph_{\alpha}, \aleph_{\alpha}\right)$ has property $E$ and $\omega_{\alpha}$ is cofinal with $\omega_{0}$, then $\alpha=0$ (see e.g. [8] Theorem 23.8). The proof of Theorem 6 uses Theorem 2 and is analogous to that of Theorem 5 .

TheOREM 6. Suppose that $X$ is an $(\alpha, \beta)$-space, $\alpha \geq \beta$, $\left(\aleph_{\alpha^{\prime}}, \aleph_{\alpha^{\prime}}\right)$ has property $E, B$ is the union of $\aleph_{\alpha^{\prime}}$ closed sets in $X$ and either

(i) $\beta$ is cofinal with $\omega_{0}, \alpha$ is not cofinal with $\omega_{0}$, and $\alpha=\alpha^{\prime}$; or

(ii) $\beta$ is cofinal with $\omega_{0}, \alpha$ is cofinal with $\omega_{0}$, and $\alpha^{\prime}=\alpha+1$; or

(iii) $\alpha>\beta+\omega_{0}, \alpha$ is not cofinal with $\omega_{0}$, and $\alpha^{\prime}=\alpha$; or

(iv) $\alpha \geq \beta+\omega_{0}, \alpha$ is cofinal with $\omega_{0}$, and $\alpha^{\prime}=\alpha+1$; or

(v) $\alpha<\beta+\omega_{0}$, and $\alpha^{\prime}=\beta+\omega_{0}+1$. 
Then $B$ is the image, under a continuous one-to-one function, of a complete 0 -dimensional (in any sense) metric space of weight $\leqq \aleph_{\alpha^{\prime}}$.

\section{REFERENCES}

1. P. Alexandroff, On some results concerning topological spaces and their continuous mappings, General Topology and its Relations to Modern Analysis and Algebra, Proc. Prague Sympos. 1961, 41-54, Academia, Prague, 1962.

2. A. V. Arkhangel'skii, Mappings and Spaces, Uspehi Mat. Nauk 21 (1966), no. 4 (130), 133-184; Russian Math. Surveys 21 (1966), no. 4, 115-162.

3. D. W. Bressler and M. Sion, The current theory of analytic sets, Canad. J. Math. 16 (1964), 207-230.

4. R. Engelking, Outline of general topology, North-Holland, Amsterdam, 1968.

5. J. L. Kelley, General topology, Van Nostrand, Princeton, 1959.

6. Arthur H. Kruse, Souslinoid and analytic sets in a general setting, Amer. Math. Soc. Memoir no. 86, 1969.

7. E. Michael, Representing spaces as images of metrizable and related spaces, Gen. Top. Appl. 1 (1971) 329-344.

8. J. Donald Monk, Introduction to set theory, McGraw-Hill, New York, 1969.

9. V. Ponomarev, Metric spaces and the continuous mappings connected with them, Dokl. Akad. Nauk SSSR 153 (1963), 1013-1016; Soviet Math. Dokl. 4 (1963), 1777-1780.

10. - Normal spaces as images of zero-dimensional ones, Dokl. Akad. Nauk SSSR 132 (1960), 1269-1272; Soviet Math. Dokl. 1 (1960), 774-776.

11. T. W. Rishel, Nice spaces, nice maps, General Topology and its Relations to Modern Analysis and Algebra, III. Proc. Third Prague Topological Sympos. 1971, 375-383, Academia, Prague, 1972.

12. C. A. Rogers, Descriptive Borel sets, Proc. Royal Soc., A, 286 (1965), 455-478.

13. A. K. Steiner and E. F. Steiner, The natural topology on the space $A^{B}$, J. Math. Anal. App. 19 (1967), 174-178.

14. A. H. Stone, Non-separable Borel sets, Rozprawy Matematyczne 28, Warsaw, 1962.

DEPARTMENT OF MATHEMATICS

QUEEN'S UNIVERSITY

KINGSTON, ONTARIO K7L 3N6 